\title{
From Stems (and Stars) to Roses: Shape-Controlled Synthesis of Zinc Oxide Crystals
}

Marco Palumbo , Thierry Lutz , Cristina E. Giusca, Hidetsugu Shiozawa , Vlad Stolojan, David C. Cox , Rory M. Wilson, Simon J. Henley, and S. Ravi P. Silva

Nano-Electronics Centre, Advanced Technology Institute, Faculty of Physics and Engineering Sciences, University of Surrey, Guildford GU2 7XH, United Kingdom

\section{Abstract}

A novel, scalable, solution-based method suitable for the control of $\mathrm{ZnO}$ crystal morphology at moderate temperature from rods/stars to rose-shaped, flowerlike structures is presented here. The synthesized $\mathrm{ZnO}$ roselike crystals are composed by wide nanosheets folded several times on themselves. This study represents the first step toward the full understanding of the growth mechanism that led to the formation of such a structure. It is now widely acknowledged that both size and shape of the metal oxide crystals have a profound effect on their properties. The influence that two morphology directing agents, ammonia and citric acid trisodium salt dihydrate, have on the shape-controlled synthesis is illustrated. The analysis of various possible mechanisms and forces that might contribute to the roselike crystals formation, such as the influence of polar surfaces, is discussed. The chemical composition and work function of the $\mathrm{ZnO}$ roses is investigated via XPS and UPS. XRD analysis suggests wurtzite as the most likely crystal structure for both the $\mathrm{ZnO}$ stars and roses, albeit these latter have a more elusive diffractogram because of their folded configuration. PL data suggest a lower level of interstitial oxygen for both crystals synthesized, starlike and flowerlike. Analysis of the ratio of the emission in the Blue range upon the ultraviolet emission indicates that the $\mathrm{ZnO}$ stars are slightly more conductive than the $\mathrm{ZnO}$ roses, a factor associated with the lower native defect concentration in these latter structures.

\section{Introduction}

Zinc oxide is a large direct band gap semiconductor with enormous potential applications in ultraviolet optoelectronics, room temperature lasing, transparent electronics, and power generation. (1-4) ZnO crystals and nanostructures may be grown by a variety of methods including the use of templates, chemical vapor deposition (CVD), electrodeposition, pulsed laser ablation, and solution-based processes. $(1,5,6)$ It is becoming clearer now that not only the size but also the shape of metal oxide and semiconductor crystals has a profound effect on their properties.(7) However, the mesoscopic control of hierarchically organized and nanostructured crystals, and the associated applications in the advanced materials field, is still an open challenge.(8) $\mathrm{ZnO}$ has proved over the years to have one of the richest families of 
nanostructures of all materials, with example including rods, wires, ribbons, springs, spirals, closed rings, tetrapods, cages, and combs. $(9,10)$ In this work, we will illustrate a novel, facile, scalable, solution-based method suitable for the tuning of $\mathrm{ZnO}$ crystal morphology at moderate temperature from rods/stars to roselike structures, composed by wide nanosheets folded several times on themselves.

The method exploited in this study is based on an inexpensive aqueous solution synthesis procedure first proposed for the growth of $\mathrm{ZnO}$ nanorods onto planar substrates, i.e., hydrothermal decomposition of precursors at moderate temperature.(11) Solution-based methods are generally simpler and less expensive for the production of nanomaterials than other techniques. Furthermore, when water is used as the solvent, the environmental impact of the synthesis is notably reduced. Compared to the original work of Vayssieres,(11) our crystal growth is based on the control of the reactant concentration far from the equimolar equilibrium, and the introduction of citric acid trisodium salt dihydrate, $\mathrm{HOC}(\mathrm{COONa})\left(\mathrm{CH}_{2} \mathrm{COONa}\right)_{2} \cdot 2 \mathrm{H}_{2} \mathrm{O}$, to alter the preferential plane of adsorption of the ions in solution. In the present configuration, there is no use of a substrate or template to guide the crystal growth. The fundamental principle of the hydrothermal synthesis of $\mathrm{ZnO}$ rods is based on the release of zinc and oxygen from the thermal decomposition of specific reactants and the building-up of a wurtzite crystal along the c-axis. From the point of view of the growth, then, the polar facets present preferential adsorption sites both for the Zinc and for the Oxygen ionic species. $(5,11)$ In this specific case, zinc nitrate hexahydrate, $\mathrm{Zn}\left(\mathrm{NO}_{3}\right)_{2} \cdot 6 \mathrm{H}_{2} \mathrm{O}$, and hexamethylenetetramine, HMTA $\left(\mathrm{CH}_{2}\right)_{6} \mathrm{~N}_{4}$, were employed as precursors. In contrast with earlier work, (5) the concentration of HMTA was 1 order of magnitude higher than that of zinc nitrate. Already, this alteration produced quite dramatic outcomes in contrast with the equimolar case. In fact, the shapes and dimensions of the $\mathrm{ZnO}$ crystals were quite varied, with a range of forms that went from rods of different lengths and width, to starlike formations. On the other end, the addition of sodium citrate brought a remarkable uniformity in the synthesis outcome: large $\mathrm{ZnO}$ nanosheets folded on themselves several times to produce flowerlike crystals, hereafter referred to as $\mathrm{ZnO}$ nanoroses. Dimensions and shapes were consistent through the samples analyzed (i.e., within the same synthesis run or between different runs). It is also important to note that in the latter case, $\mathrm{ZnO}$ nanoroses were the only crystal shape produced. Previously, cathodic electrodeposition has been used for the growth on polycrystalline $\mathrm{Zn}$ foil of $\mathrm{ZnO}$ films of various morphologies from 1D (nanorods), 2D (nanoplates) to 3D crystals. $(12,12)$ In that study, however, the two-dimensional sheet growth-50 nm in thickness and several micrometers in diameter-was found to occur only in 
conjunction with the nanorods under specific electrochemical conditions, indicating that their formation mechanism was related to the one controlling the $\mathrm{ZnO}$ nanorod growth.(12)

\section{Experimental Section}

Zinc nitrate hexahydrate, $\mathrm{Zn}\left(\mathrm{NO}_{3}\right)_{2} \cdot 6 \mathrm{H}_{2} \mathrm{O}$, and hexamethylenetetramine, $\left(\mathrm{CH}_{2}\right)_{6} \mathrm{~N}_{4}$, solutions were prepared in ultrapure water. Molar concentration were typically $1 \times 10^{-3} \mathrm{M}$ for the zinc nitrate and $1 \times 10^{-2} \mathrm{M}$ for the hexamethylenetetramine. For the synthesis of the $\mathrm{ZnO}$ flowers, 2 $\times 10^{-4} \mathrm{M}$ citric acid trisodium salt dehydrate, $\mathrm{HOC}(\mathrm{COONa})\left(\mathrm{CH}_{2} \mathrm{COONa}\right)_{2} \cdot 2 \mathrm{H}_{2} \mathrm{O}$, was added to the original solution. The measured $\mathrm{pH}$ of the final solution before placing the container in the oven was around 6.3, whereas the $\mathrm{pH}$ at the end of the synthesis was measured at 9.5. The sodium citrate concentration indicated above was found to be ideal for the growth of the $\mathrm{ZnO}$ nanoroses. When, for example, a concentration of 2 orders of magnitude of citric acid was used, no crystal growth was observed, probably because of the resulting highly acidic environment. After vigorous stirring, the solutions were transferred to screw cap glass laboratory bottles. The bottles were then loaded inside a vacuum oven. The pressure inside the oven was close to or lower than $0.5 \mathrm{~atm}$ and the temperature was set up to $80{ }^{\circ} \mathrm{C}$ overnight. In a counter experiment, we used a normal oven instead of a vacuum one with similar results. Limited to the pressure value used in this series of experiments, no immediate conclusions can be therefore drawn on the role of the vacuum pressure on the crystal growth and on the obtained shapes. The temperature inside the bottle is assumed in first instance to be in equilibrium with the set temperature of the oven given the time necessary for the growth to be completed. We have measured that to observe the clear formation of $\mathrm{ZnO}$ nanoroses the solution has to be placed in the oven for at least $6 \mathrm{~h}$. Normally, we left the synthesis to run overnight to consume the maximum quantity of precursors possible. However, no clear change in shape or structure was observed beyond the above-mentioned $6 \mathrm{~h}$. ZnO crystals were separated from excess reagents or secondary products via centrifugation, washed in ultrapure water, dried in oven, and then resuspended in isopropanol. A Hitachi HD2300A scanning trasmission electron microscope was used (200 keV acceleration voltage, Schottky field emission source) to analyze the ZnO flowerlike crystals. The probe diameter was $2.5 \AA$ and spot diffraction was performed with a very thin $(\sim 3 \mathrm{~nm})$, nearly parallel beam. Focused ion beam cross-sectioning was carried out on a FEI Nova Nanolab 600 dual-beam FIB. An ion beam current of 300pA was used and the ion milling was observed in real time with the electron column on the same instrument, using $10 \mathrm{kV}$ and $3 \mathrm{nA}$ of beam current. The $\mathrm{ZnO}$ roses were dispersed on a standard 300 mesh copper TEM grid which was in turn mounted on the FIB stage. Several $\mathrm{ZnO}$ roses were sectioned and all were found to contain a hollow core. X-ray 
powder diffraction was carried out on a Panalytical X'pert Pro diffractometer using an X-ray tube operated at $45 \mathrm{kV}$ and $40 \mathrm{~mA}$ with a copper target and Ni filter giving Cu Ka1 and Ka2 radiation. The samples were mounted as a dusting on a silicon single crystal slice, cut so that it will not produce any crystalline diffraction with copper wavelengths, and rotated continuously, in the sample plane, to improve the powder average. Data was collected over the range $5-120^{\circ} 2 \theta$ with the Panalytical X'celerator detector operating in continuous mode. An Omicrometer Multiprobe UHV system equipped with an Omicrometer EA125 hemispherical analyzer was used for both XPS and UPS experiments. For XPS, spectra were acquired with a pass energy of 50 and $20 \mathrm{eV}$ for survey and higher resolution respectively, using $\mathrm{Mg} \mathrm{Ka}$ radiation $(h v=1256.4 \mathrm{eV})$ from a VG XR3E2 twin anode source. The base pressure was in the range of $1 \times 10^{-9} \mathrm{mbar}$ for the entire set of experiments. The $4 \mathrm{f} 7 / 2$ line of an Au substrate was used for energy calibration. For UPS, He I (21.22 eV) from an Omicrometer HIS13 windowless He lamp was used. The pass energy was set to $5 \mathrm{eV}$ for a $6 \mathrm{~mm}$ diameter analysis area defined by the entrance slit to the analyzer. Fluorescence spectra were recorded using a xenon lamp spectrophotometer. A monochromator and a filter were used to isolate the excitation wavelength of $300 \mathrm{~nm}$.

\section{Results and Discussions}

In Figure 1 and 2, examples of starlike and flowerlike $\mathrm{ZnO}$ crystals are shown respectively. The dramatic difference in shape and morphology is evident. Describing with accuracy the crystal formation in these two cases is particularly complex, given the nature and number of the reactants involved, their concentration, and the secondary products and complex ions in solution that contribute to the kinetics of the crystal growth.
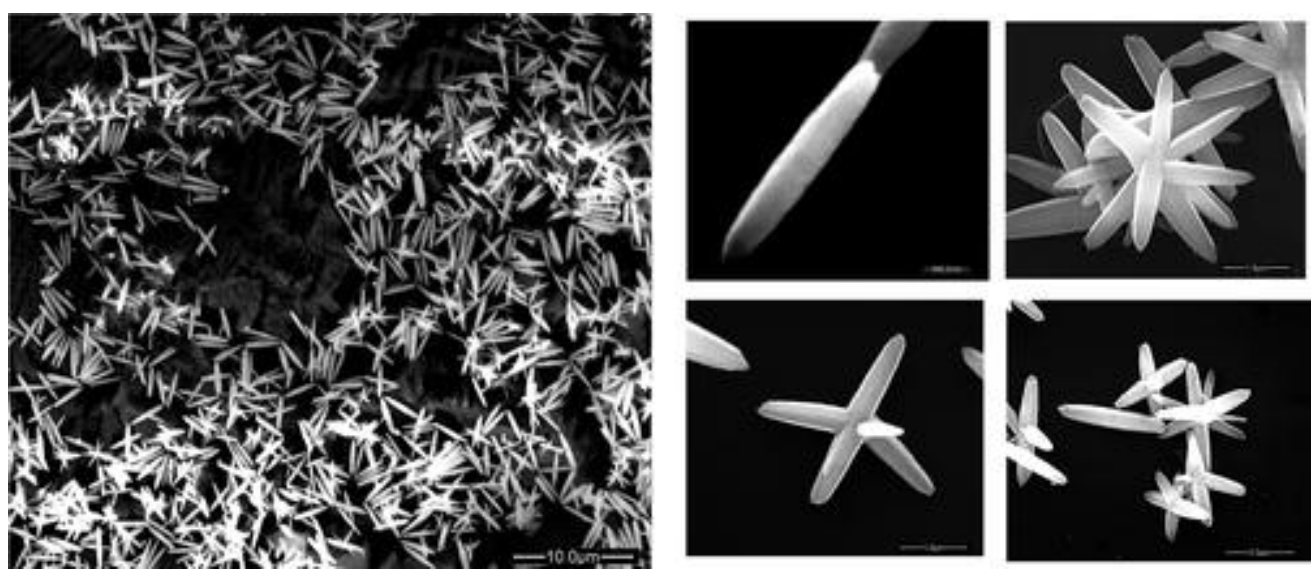

Figure 1. SEM images of the $\mathrm{ZnO}$ starlike crystals. 
A)

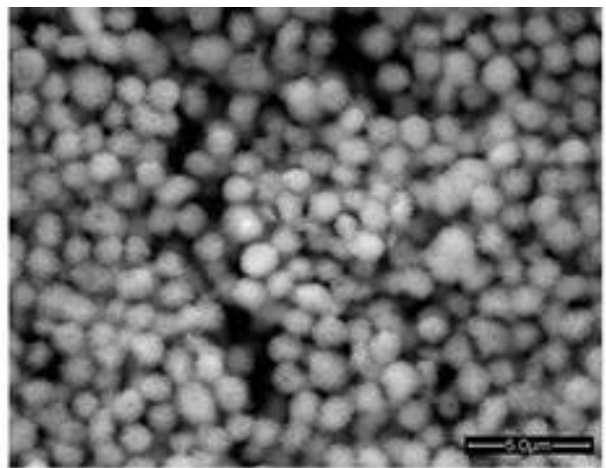

B)

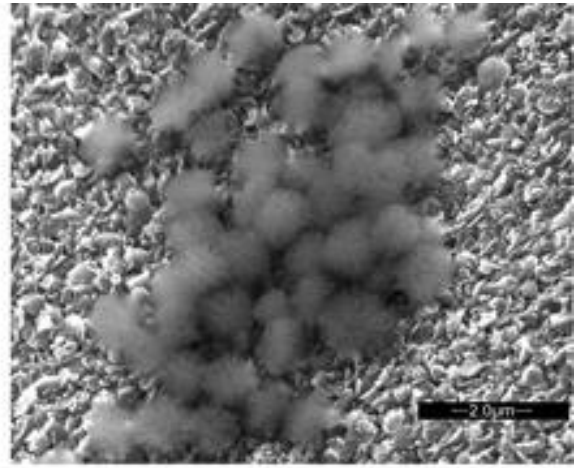

C)

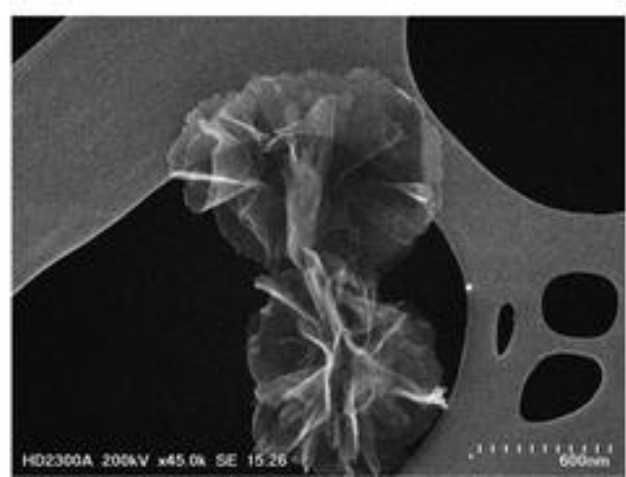

Figure 2. (A, B) Low-resolution SEM images of $\mathrm{ZnO}$ roses deposited by drop casting on a solid support. In B, the substrate was a AZO coated glass slide, courtesy of Dr. Wijayantha, Loughborough University. (C) Secondary electron, SE image of ZnO roses crystals obtained by STEM. The ZnO roses are placed over a TEM copper grid covered by a carbon membrane.

(A) ZnO Stars. An important factor to consider is that the concentration of $\left(\mathrm{CH}_{2}\right)_{6} \mathrm{~N}_{4}$ is 1 order of magnitude higher than $\mathrm{Zn}\left(\mathrm{NO}_{3}\right)_{2} \cdot 6 \mathrm{H}_{2} \mathrm{O}$. At first sight, this might suggest the production of an excess of oxygen containing species in solution. As a consequence, there should be an increased competition for the interaction with a limited number of Zinc ions. One would expect then a reduction in the size of the $\mathrm{ZnO}$ rods compared to those hydrothermally formed from an equimolar reactants solution.(5) However, despite this preface, we did not observe any apparent reduction in size. When in rod form, the $\mathrm{ZnO}$ crystals have sharp ends, a phenomenon attributed to a transition from two-dimensional to one-dimensional growth, when the concentration of free zinc and free oxygen in solution diminishes. (5) A high number of starlike crystals was also observed. A more in-depth analysis of the chemical reactions in solution reveals that there is in effect an excess of ammonia produced from the thermal decomposition of HMTA. Ammonia itself has been reported as a morphing agent for the production, in a basic environment, of $\mathrm{ZnO}$ crystals composed of several "swords" rooted on each plane of a central hexagonal cone.(13) The key aspect of this process is the ratio of the two sources of $\mathrm{Zn}$ in solution: $\mathrm{Zn}(\mathrm{OH})_{2}$ and $\left(\mathrm{Zn}\left(\mathrm{NH}_{3}\right)_{4}\right)^{2+}$ with primary and secondary nucleation stages and crystal growth triggered at higher $\mathrm{pH}$ values when the complex of $\mathrm{Zn}$ and Ammonia 
dissociates into free $\mathrm{Zn}^{2+}$ ions and then is transformed into $\left(\mathrm{Zn}(\mathrm{OH})_{4}\right)^{2-}$ growth units. A similar effect should then be responsible for the formation of the star-shaped $\mathrm{ZnO}$ in our sample.

(B) ZnO Roses. As shown in Figure 2, the effect of citric anions released from the dissociation of the sodium citrate in water has dramatically altered the growth mechanism of the $\mathrm{ZnO}$. Liu et al. have shown that citrate can be effective in creating complex $\mathrm{ZnO}$ film morphologies.(14)In particular, the height/width of $\mathrm{ZnO}$ nanorods formed by seeded growth on a substrate was found to rapidly decrease with the increasing citrate concentration. It was found that for a concentration close of above $1.2 \mathrm{mg} / 30 \mathrm{~mL}$, the rods were formed by a continuous stacking of platelets one on top of another. By secondary or tertiary growth in the presence of citrate, from oriented $\mathrm{ZnO}$ nanorods as starting material, complex superstructures composed by nanocolumns and nanoplatelets were formed. Citrate anions will selectively adsorb on $\mathrm{ZnO}$ basal planes, acting as structure-directing agents, inducing a significant modification of the surface properties and mineral growth behavior. Because the organic molecules will selectively adsorb on the (0001) surfaces, this will inhibit the crystal growth along the $\langle 0001\rangle$ orientation. Contrasted with the $\mathrm{ZnO}$ Stars case, the introduction of citric acid trisodium salt dihydrate in solution adds an element of complexity in the crystal growth. In fact, we have now a second adsorbate and a third $\mathrm{Zn}$ source in the form of $\left(\mathrm{Zn}\left(\mathrm{C}_{6} \mathrm{H}_{5} \mathrm{O}_{7}\right)_{4}\right)^{10-}$. When citric acid is used alone, the hydrothermal growth dynamics are reported as following: at first, a large quantity of $\mathrm{ZnO}$ nuclei are formed from the decomposition of $\mathrm{Zn}(\mathrm{OH})_{2}$; however, some of the zinc hydroxide will form $\left(\mathrm{Zn}\left(\mathrm{OH}_{4}\right)\right)^{2-}$ under alkaline conditions. The polar growth of $\mathrm{ZnO}$ along the $\langle 0001\rangle$ direction proceeds through the adsorption of $\left(\mathrm{Zn}_{(}\left(\mathrm{OH}_{4}\right)\right)^{2-}$ onto the $(0001)$ plane. Crucially, $\left(\mathrm{Zn}\left(\mathrm{C}_{6} \mathrm{H}_{5} \mathrm{O}_{7}\right)_{4}\right)^{10-}$ as well will preferentially adsorb onto the same crystallographic plane, thus hindering the adsorption of $\left(\mathrm{Zn}\left(\mathrm{OH}_{4}\right)\right)^{2-}$. This substantially suppresses the anisotropic growth of $\mathrm{ZnO}$ along the $(0001\rangle$ direction.(13) In the formation of the flowerlike $\mathrm{ZnO}$ presented in this paper, the two molecules (i.e., ammonia and citric anions) are both present in solution and this, as stated, is bound to alter even further the growth dynamics. Considering the data accumulated to this point, it seems reasonable to speculate that the formation of the extremely thin and extended $\mathrm{ZnO}$ nanosheets that form the flowers is guided by the interaction and contribution of all the different $\mathrm{Zn}$ sources in solution, and, it is linked to acid-alkaline transitions in solution. Other parameters, such as temperature growth and precursor concentration, are very likely to play a role as well(15, 16) and further research is necessary to fully understand their influence. In our study, we did not observe any remarkable variation in the crystal growth by varying the oven temperature between 80 and $90{ }^{\circ} \mathrm{C}$, very likely because of the synthesis being run mostly overnight and, in 
any case, for more than $6 \mathrm{~h}$, therefore leveling the effect of temperature on the synthesis growth rate.

A further element useful for the understanding of the $\mathrm{ZnO}$ flowers structure comes from the milling of these crystals by a focused gallium ion beam. The $\mathrm{ZnO}$ crystals were tilted with respect to the horizontal plane and the beam focused to impact on a plane close to the flowers to be able to effectively slice them layer by layer. As can be appreciated from Figure 2, the $\mathrm{ZnO}$ crystals appear to be mesoporous structures similar to precedent polyhedral cages and shells formed by textured self-assembly $\mathrm{ZnO}$ nanocrystals synthesized via a solidstate thermal sublimation process.(10) In that specific study, the shell/cage formation started from a central metallic Zinc cluster to then progress via oxidation of the original nucleus with consequent migration of the $\mathrm{Zn}$ ions toward the outer surface to leave an empty core behind. Such a mechanism appears to be unlikely to happen in our case, because of the high oxidizing environment in which the growth takes place. In some studies, the formation of $\mathrm{ZnO}$ sheets and plates from a vapor-liquid-solid procedure has been attributed to a 1D branching and subsequent 2D interspace filling process (sheets) to give a final 3D structure (plates).(17) In our case, a more credible hypothesis is that the nanosheets fold while growing, similarly to crepe paper flowers. The nanosheets might be the result of the self-assembly of a number of nanocrystals that come together to form a hierarchical mesostructure (see later in Figure 3discussion). The final outcome of this process is a complex three-dimensional architecture which includes internal empty volumes.

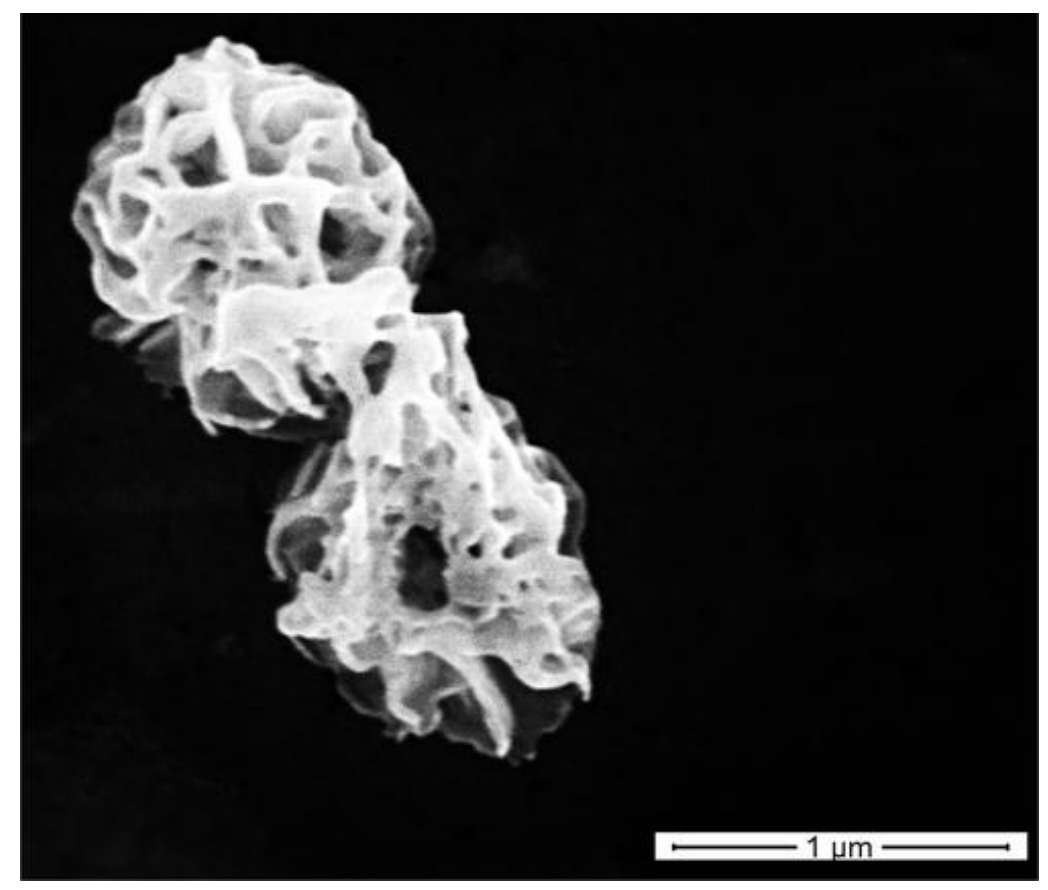


Figure 3. SEM image of $\mathrm{ZnO}$ nanoroses that have been milled by a focused gallium ion beam. The internal porous structure is now evident.

Considering the large curvature ratio the $\mathrm{ZnO}$ nanosheets are subject to, their strength and flexibility is notable. The folding itself of the nanosheets recalls an analysis of the different forces involved in the process. In primis, (I) polar charges from the oppositely charged $\left(\mathrm{Zn}^{2+}\right.$, $\mathrm{O}^{2-}$ ) basal planes of the $\mathrm{ZnO}$ crystal will tend to compensate each other to give a minimum energy configuration. However, (II) electrostatic-induced elastic deformations may also play a role, albeit opposite to that of the polar surfaces, toward the attainment of such a minimum energy state.(9) In the present study, the surface charges may partially be screened by the complex ions in solution. Nevertheless, if unbalanced, these, together with the induced elastic strain, could end up being the driving force of the sheets folding, with the subsequent formation of short-range forces, i.e., chemical bonds, to give a final equilibrium structure. Moreover, in some cases, (III) surface tension/energies in-homogeneities between two opposite crystal surfaces might as well induce the spontaneous bending of the crystals.(18) In Figure 4, a pictorial description of a hypothetic nanosheet folding mechanism is shown. The solid arrows in Figure $4 \mathrm{~A}$ indicate the forces that drive the sheet formation via sequential addition of zinc and oxygen ions, whereas the arrows with a striped pattern indicates the vectorial sum of forces (e.g., electrostatic forces, induced elastic strain, short-range forces) that induce the folding. In Figure 4B, a trasmission electron (TE) image shows the final result of this process. A very thin $(-3 \mathrm{~nm})$ nearly parallel beam was used to perform spot diffraction (see Figure $4 C$ ). The very thin $\mathrm{ZnO}$ nanosheets do suffer exposure to the beam, as it can be appreciated by the number of small holes, drilled through the $\mathrm{ZnO}$ flower shown in Figure 4B. As a consequence, the collected diffraction pattern gives us only a limited amount of information about the crystal nature of the $\mathrm{ZnO}$ flowers. 


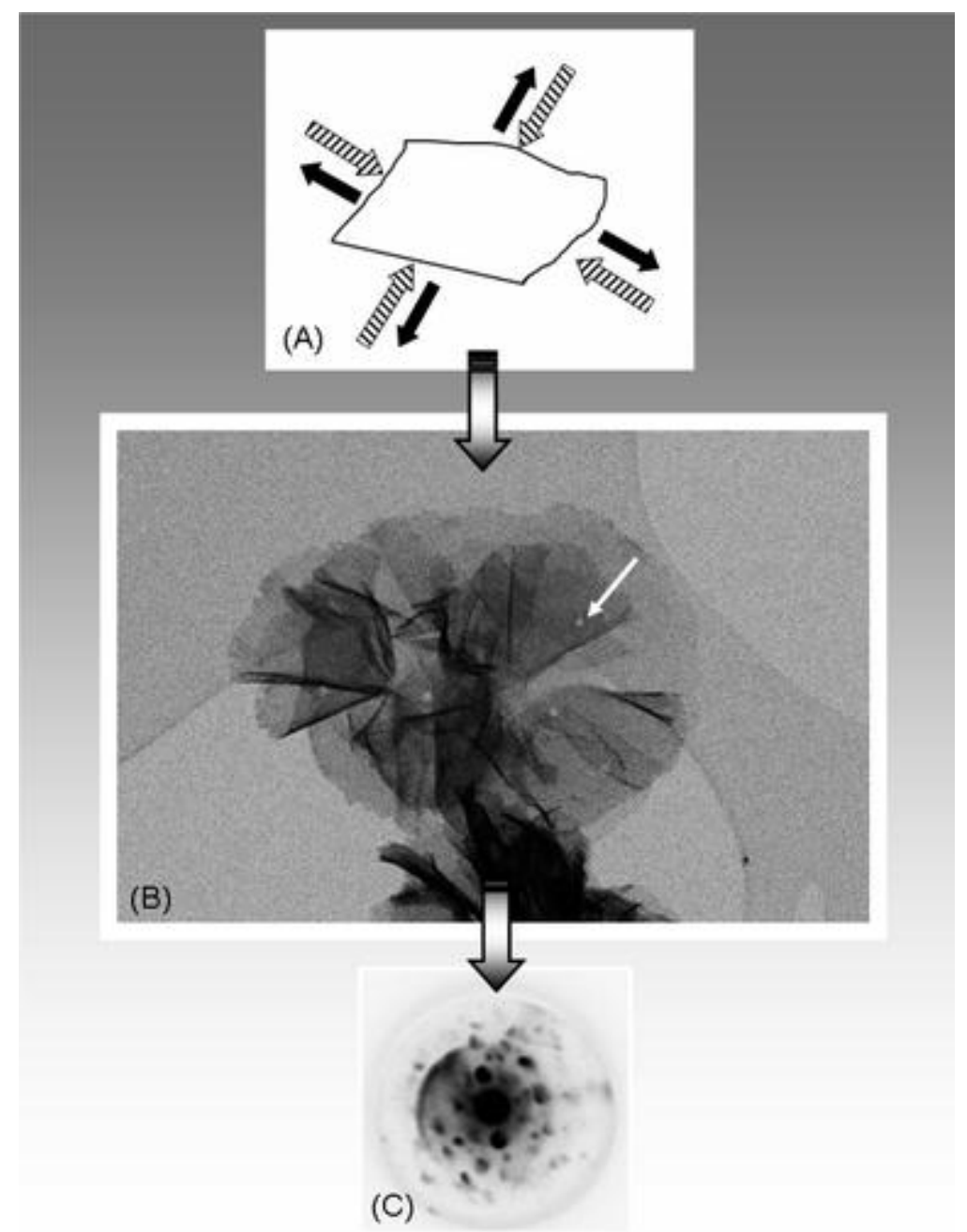

Figure 4. (A) Proposed folding mechanism of a ZnO nanorose; (B) TE image of the resulting flowerlike crystal. The arrow indicates one of the holes drilled by the beam during the acquisition of a diffraction pattern; (C) diffraction pattern showing the crystalline nature of the $\mathrm{ZnO}$ flower.

To further characterize the synthesized crystals, we then decided to collect additional X-ray diffraction spectra. In Figure 5, we show from the bottom to the top of the figure, first the indexed RRUFF (from http://rruff.info) XRD diffractogram for wurzite ZnO (R050492-1), also known as zincite, and then the experimental XRD spectra from a sample of $\mathrm{ZnO}$ starlike crystals and $\mathrm{ZnO}$ roses. (For the zincite RRUFF diffractogram we have indexed the pattern.) Contrasting the data in the literature with our experimental XRD data brings us to the conclusion than the synthesized $\mathrm{ZnO}$ starlike crystals have a hexagonal wurzite crystallographic structure with an almost perfect match with the zincite RRUFF data. For the $\mathrm{ZnO}$ roses, the identification of a clear crystalline structure is more uncertain, which is likely to be due to the complex folded structure and perhaps to a poly nanocrystalline nature as previously found for the $\mathrm{ZnO}$ polyhedral mesopouros cages.(10) In this case, the match with the indexed RRUFF zincite spectra is limited to five peaks: $100,002,101,110$, and 103 . In addition to this, we 
notice two additional broad peaks on the right side of 100 and 110 that might be due to some level of disorder in the crystals, perhaps driven by the complex folding of the nanosheet.

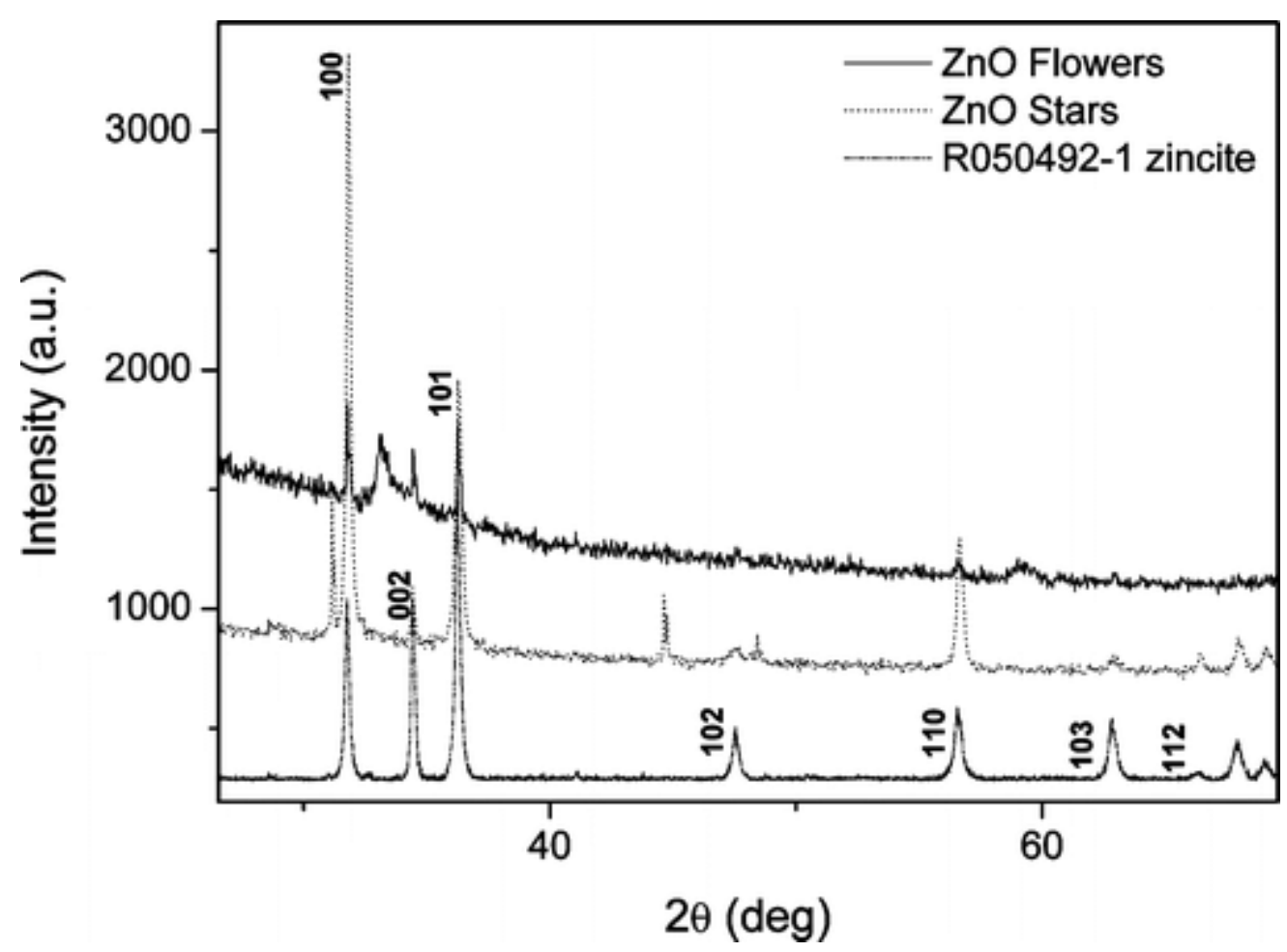

Figure 5. XRD spectra for indexed $\mathrm{ZnO}$ wurzite, $\mathrm{ZnO}$ stars, and $\mathrm{ZnO}$ nanoroses.

Information on the chemical composition of the $\mathrm{ZnO}$ flowers and the valence band structure of the crystals were obtained via X-ray photoelectron spectroscopy, (XPS) and ultraviolet photoelectron spectroscopy, (UPS). A thin layer of ZnO flowers was prepared on a support coated with $100 \mathrm{~nm}$ of Au deposited via thermal evaporation. To obtain a uniform coating, we achieved a slow evaporation of the solvent by placing the support in an environment saturated with the same solvent vapors, a technique already exploited, for example, for the uniform deposition of semiconductor quantum dots.(19) XPS survey scans indicated the presence of both $\mathrm{Zn}$ and $\mathrm{O}$ in the sample, for which high resolution scans of the $\mathrm{Zn} 2 \mathrm{p}$ and $\mathrm{O}$ 1s regions were recorded. In Figure 6A, the XPS spectra of $Z n 2 p_{3 / 2}$ and $Z n 2 p_{1 / 2}$ peaks before and after annealing in ultrahigh vacuum (UHV) at $250{ }^{\circ} \mathrm{C}$ for $3 \mathrm{~h}$ are shown. The main peaks located at 1025 and $1048 \mathrm{eV}$ can be assigned to the $\mathrm{ZnO}$ crystals. The energy difference of these two peaks agrees well with the standard value of $22.97 \mathrm{eV}(20)$ and no major shift of the binding energies is observed at the end of the annealing process. More importantly, we notice the energy peaks at 1021 and $1044 \mathrm{eV}$ corresponding to elemental $\mathrm{Zn}$ in both spectra. They can be associated with interstitial zinc within the $\mathrm{ZnO}$ crystal, which is relevant for its photoluminescence and conductive properties, as will be discussed later in the text. The 
stochiometric ratio of $\mathrm{ZnO}$ to $(\mathrm{Zn}+\mathrm{ZnO})$ is close to $90 \%$ for both cases. In Figure $6 \mathrm{~B}$, the normalized relative atomic ratio between $\mathrm{Zn}$ and $\mathrm{O}$ for three different annealing stages is shown. We associate the drop in oxygen content, from ca. 60 to $44 \%$ after $1 \mathrm{~h}$ at $250{ }^{\circ} \mathrm{C}$ and further down to ca. $43 \%$ after $3 \mathrm{~h}$, to the removal of "atmospheric" oxygen and water adsorbed on the outer surface of the $\mathrm{ZnO}$ roses prior to the introduction to the UHV chamber. The apparent increase in Zinc content is a consequence of the normalization process of the experimental data. The measured binding energy of oxygen $1 \mathrm{~s}$ at the different stages of the annealing process shifts toward lower binding energy, from $535.5 \mathrm{eV}$ for the pristine $\mathrm{ZnO}$ flowers to $534.75 \mathrm{eV}$ after one hour of annealing, a value kept constant until the end of the annealing process. The work function of the $\mathrm{ZnO}$ crystals was determined from the UPS on the annealed sample. The sample was biased at $-3 \mathrm{~V}$ during the work function measurements in order to accelerate the low energy secondary electrons. The work function was determined directly from the low kinetic energy cutoff of the UPS spectra. The work function of the underlying gold substrate was calculated to be $4.7 \mathrm{eV}$, whereas the work function of the $\mathrm{ZnO}$ flowers was found to be close to 3.2.eV. 

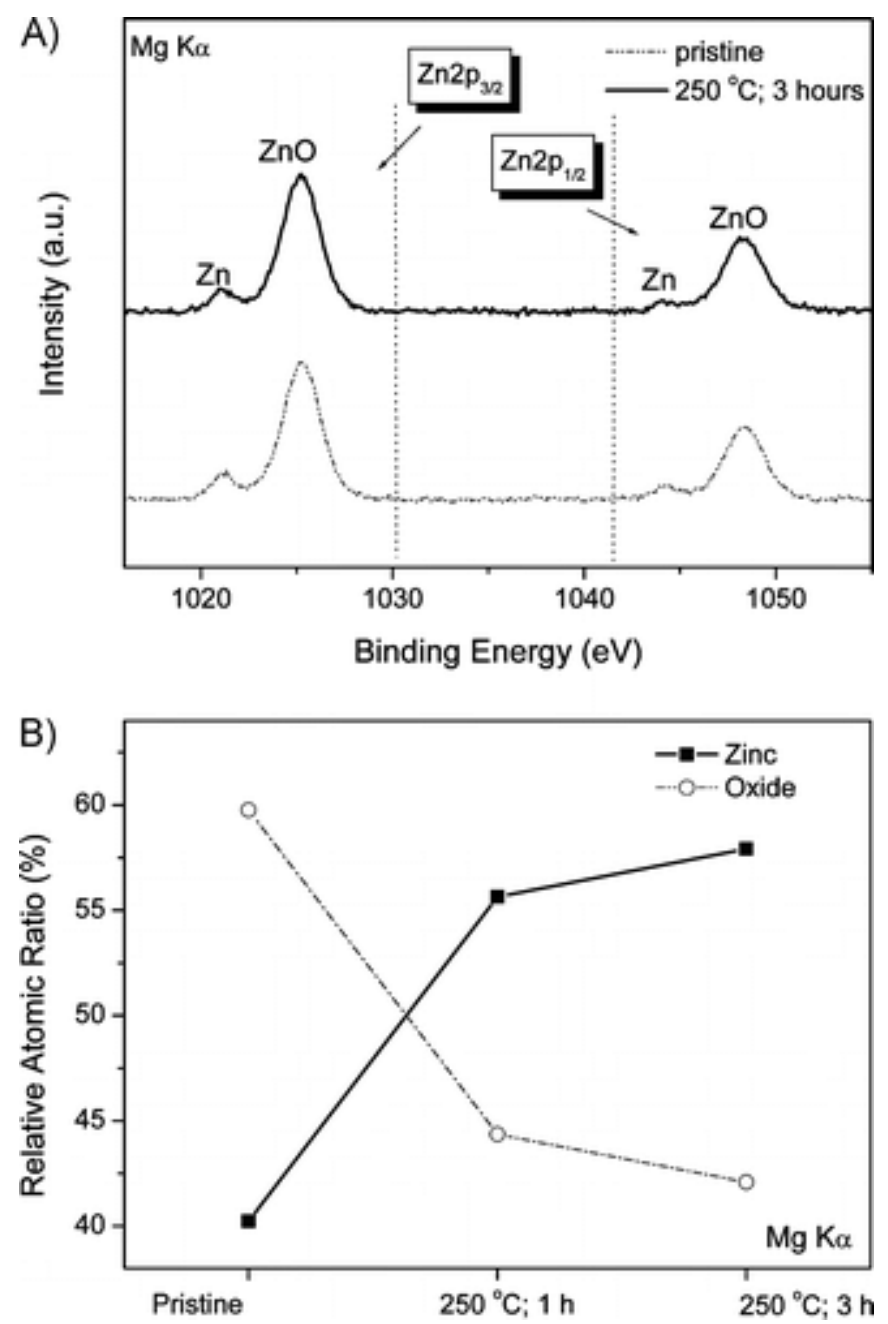

Figure 6. (A) XPS spectra of the ZnO nanoroses before and after annealing for 3 hours at $250^{\circ} \mathrm{C}$. (b) Normalized relative atomic ratio for zinc and oxygen at various stages of the annealing process.

Finally, photoluminescence spectra like those shown in Figure 7 can be useful for understanding the nature of the intrinsic defect in the synthesized $\mathrm{ZnO}$ because the energy levels associate with the defects populating the large band gap of the material and producing radiative emission at different wavelengths.(5) The first emission peak as shown in Figure 7 is the near band edge peak in the ultraviolet range, UVL ( $387 \mathrm{~nm}$ for the $\mathrm{ZnO}$ flowers, whereas the UVL for the ZnO stars is slightly red-shifted at $395 \mathrm{~nm}$, as can be seen from the inset in the top right corner of Figure 7) and is generally attributed to interstitial zinc, $\mathrm{Zn}_{\mathrm{i}}$, in the crystal lattice. The second peak in the blue wavelength BL (at $410 \mathrm{~nm}$ for both crystals) is instead normally linked to oxygen voids, $\mathrm{V}_{0}^{-}$, where the minus sign over the oxygen symbol identifies the ionization degree of the same according the Kroger-Vink formalism.(21) The intensity ratio $\mathrm{BL} / \mathrm{UV}$ goes from 0.95 for the $\mathrm{ZnO}$ stars crystals to 0.7 for the $\mathrm{ZnO}$ flowers. The emission in the visible spectrum is generally attributed to interstitial oxygen, $O_{i}$ (see ref 5 and references 
thereafter). In the case reported, this emission is very tenuous for the $\mathrm{ZnO}$ stars and practically nonexistent for the $\mathrm{ZnO}$ roses. This fact gives us an indication of the defect level and nature within the synthesized crystals. The ratio $(B L+G L) / U V L$, where $G L$ is the green luminescence, has been taken into account as indication of the electrical conduction characteristic for nondoped $\mathrm{ZnO}$ nanostructures, where the conduction is entirely dependent on the native crystal defects.(5)In our case, we can assume $G L$ to be negligible at least at room temperature, a fact indeed expected.(5) Therefore, the previously calculated BL/UVL ratio can be considered instead. From this simple consideration of the PL spectra, we can deduce that nondoped $\mathrm{ZnO}$ stars are slightly better electrical conductors than $\mathrm{ZnO}$ roses, given the lower level of native defects (in particular interstitial oxygen, $\mathrm{O}_{\mathrm{i}}$ ) in the latter.

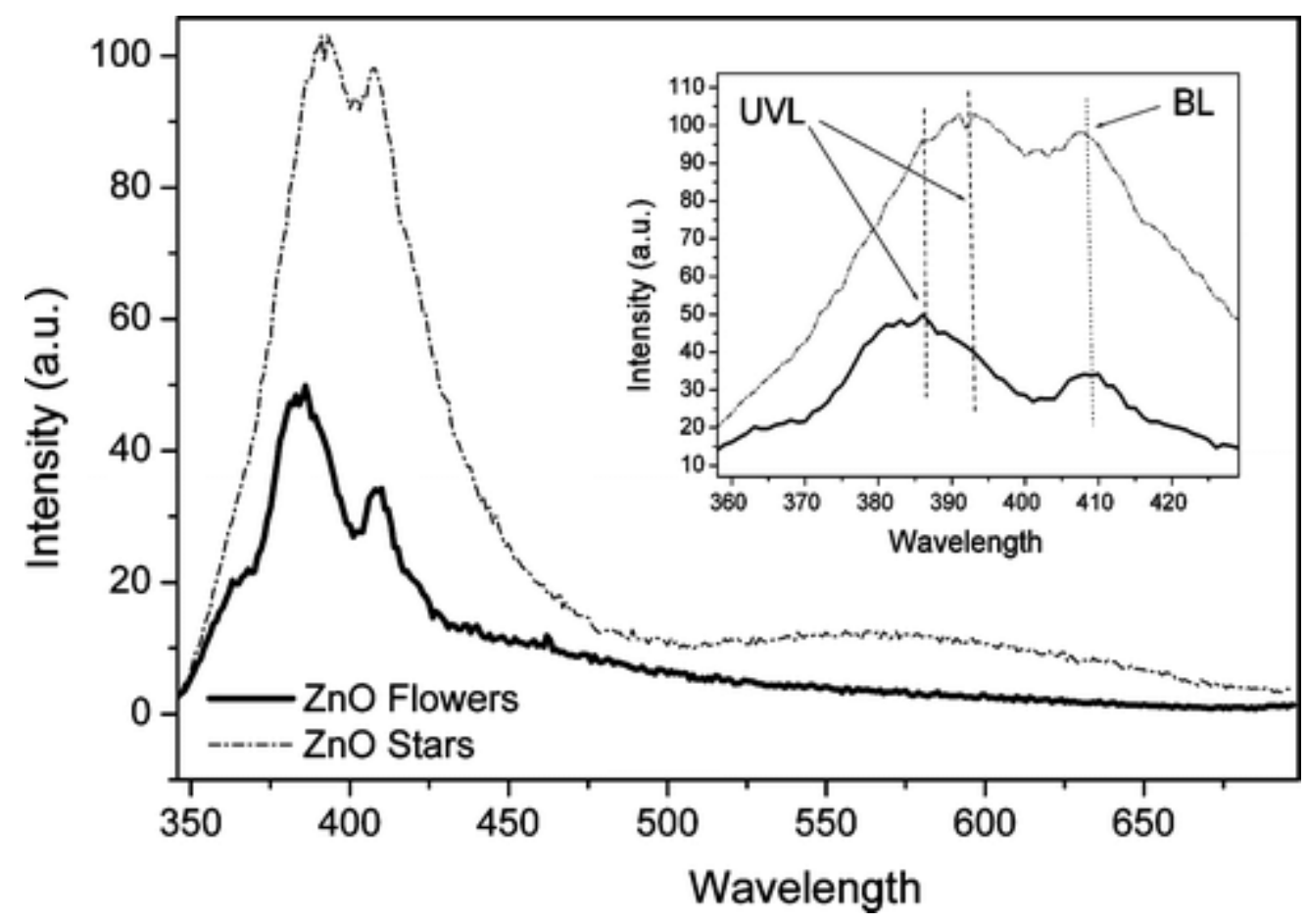

Figure 7. PL spectra for the starlike crystals and the ZnO nanoroses. Inset: close zoom on the UVL and BL peaks.

\section{Conclusions}

In conclusion, a shape-controlled solution-based method for the large-scale synthesis at moderate temperature of zinc oxide crystals was presented. The influence that two morphology directing agents, ammonia and citric acid, have on the shape-controlled synthesis was illustrated. This study augurs well for future precise engineering of $\mathrm{ZnO}$ crystals via control of their size/shape for tailored technological applications such as photocatalysis or 
photolectrochemical cells. In particular, a roselike $\mathrm{ZnO}$ crystal formed by the folding of a selfassembled $\mathrm{ZnO}$ nanosheet was introduced. Such a structure represents a first in the already large family of $\mathrm{ZnO}$ nanostructures. The analysis of various possible mechanisms and forces that might contribute to the roselike crystal formation, such as the polarization of the resulting $\mathrm{ZnO}$ crystal, was discussed. XPS analysis suggests that the crystals contain interstitial Zinc with a ratio $\mathrm{ZnO} / \mathrm{Zn}$ close to $90 \%$. XRD diffractograms show the crystal structure of the $\mathrm{ZnO}$ star to be almost coincident with that of wurzite, whereas that for the $\mathrm{ZnO}$ flowers is more difficult to analyze, given the folded nature of the nanosheets. PL data suggest a lower level of interstitial oxygen for both crystals synthesized, starlike and flowerlike. Analysis of the ratio of the emission in the blue range upon the ultraviolet emission indicates that the $\mathrm{ZnO}$ stars are slightly more conductive than the $\mathrm{ZnO}$ flowers, a factor directly correlated with the lower native defect concentration in the latter.

\section{Acknowledgment}

The authors thank EPSRC for a Portfolio Partnership award that has helped enable this research.

\section{References}

1.

Ozgur, U.; Ya, I. A.; Liu, C.; Teke, A.; Reshchikov, M. A.; Dogan, S.; Avrutin, V.;

Cho, S. J.; Morkoc, H. J. Appl. Phys. 2005, 98 ( 4) 041301

2.

Wang, X. D.; Song, J. H.; Liu, J.; Wang, Z. L. Science 2007, 316 ( 5821) 102- 105

3.

Wang, Z. L. Adv. Mater. 2003, 15 ( 5) 432- 436

4.

Huang, M. H.; Mao, S.; Feick, H.; Yan, H.; Wu, Y.; Kind, H.; Weber, E.; Russo, R.; Yang, P.Science 2001, 292, 1897- 1899

5.

Palumbo, M.; Henley, S. J.; Lutz, T.; Stolojan, V.; Silva, S. R. P. J. Appl.

Phys. 2008, 104 (7) 074906

6.

Henley, S. J.; Ashfold, M. N. R.; Cherns, D. Surf. Coat. Technol. 2004, 177-178) 271-276 
7.

Jun, Y. W.; Choi, J. S.; Cheon, J. Angew. Chem., Int. Ed. 2006, 45, 3414- 3439

8.

Zhang, Y. Y.; Mu, J. Nanotechnology 2007, 18 ( 7) 6

9.

Wang, Z. L.; Kong, X. Y.; Ding, Y.; Gao, P. X.; Hughes, W. L.; Yang, R. S.; Zhang, Y. Adv. Funct. Mater. 2004, 14 ( 10) 943- 956

10.

Gao, P. X.; Wang, Z. L. J. Am. Chem. Soc. 2003, 125 ( 37) 11299- 11305

11.

Vayssieres, L. Adv. Mater. 2003, 15 ( 5) 464- 466

12.

Illy, B.; Shollock, B. A.; MacManus-Driscoll, J. L.; Ryan, M.

P. Nanotechnology 2005, 16 ( 2)320- 324

13.

Zhang, H.; Yang, D.; Li, D.; Ma, X.; Li, S.; Que, D. Cryst. Growth Des. 2005, 5 ( 2) 547550

14.

Tian, Z. R. R.; Voigt, J. A.; Liu, J.; McKenzie, B.; McDermott, M. J.; Rodriguez, M. A.;

Konishi, H.; Xu, H. F. Nat. Mater. 2003, 2 ( 12) 821- 826

15.

Tak, Y.; Yong, K. J. Phys. Chem. B 2005, 109 ( 41) 19263- 19269

16.

Ahsanulhaq, Q.; Kim, J. H.; Hahn, Y. B. Nanotechnology 2007, 18, 485307

17.

Park, J. H.; Choi, H. J.; Choi, Y. J.; Sohn, S. H.; Park, J. G. J. Mater.

Chem. 2004, 14 ( 1)35- 36

18.

Cahn, J. W.; Hanneman, R. E. Surf. Sci. 1964, 1 ( 4) 387- 398

19.

Rizzo, A.; Mazzeo, M.; Palumbo, M.; Lerario, G.; D’Amone, S.; Cingolani, R.;

Gigli, G. Adv. Mater. 2008, 20 ( 10) 1886- 1891

20.

Moulder, J. F.; Stickle, W. F.; Sobol, P. E.; Bomben, K. D. Handbook of X-ray

Photoelectron Spectroscopy; Chastain, J.; King, R. C., Jr., Eds.; Perkin-Elmer, Physical Electronics Division: Eden Prairie, MN, 1992. 
21.

Kroger, F. A.; Vink, H. J. J. Chem. Phys. 1954, 22 ( 2) 250- 252 